\title{
Research of Feedback Correction Method Based on Neural Network
}

\author{
Biying Zhou \\ College of Mathematics and Information Science \\ Weinan Teachers University, \\ Weinan 714000, China, \\ biyingzhou@126.com.cn
}

\begin{abstract}
In the predictive control, it is often need to obtain the model which prediction accuracy is possible higher, however, in practice controlled objects are often exist nonlinear, parameter variation, model mismatch, disturbance and other factors, there is a big error between the controlled object-based prediction model and the actual output of the object. In this paper, using neural network to improve prediction model accuracy, at the same time feedback correction method is used to compensate for the lack of neural network prediction model, simulation results show that this method has better accuracy than conventional feedback correction.
\end{abstract} model

Keywords-Neural network, Feedback correction, Prediction

\section{INTRODUCTION}

All manuscripts must be in English. These guidelines include complete descriptions of the fonts, spacing, and related information for producing your proceedings manuscripts. Please follow them and if you have any questions, direct them to the production editor in charge of your proceedings at Conference Publishing Services (CPS): Phone +1 (714) 821-8380 or Fax +1 (714) 761-1784.

Prediction model is only rough description of the dynamic characteristics of controlled object, actual controlled object often exist varies interference factors, there is a big error between model prediction and actual output. The inevitable model errors will definitely lower the control accuracy and robust performance of control system, may even lead to system instability [1]. Therefore, it is not only need to improve the accuracy of prediction model but also need to take additional necessary measures to supplement the lack of model predictions.

According to this issue, introducing error prediction, the ideological of the multi-level added prediction is proposed based on error prediction supplement models. In predictive control, the dynamics causal relationship described by predictive models can be reflected by model prediction, and uncertain factors which not included in prediction model can be described by error prediction, the introduction of prediction error can effectively compensate the affect which caused by some simplified or ignored factors in predictive control.

\section{COMMONLY USED FEEDBACK CORRECTION METHOD}

Prediction model has accuracy problem, in some extent feedback correction can overcome the model error [2,3], calibration relations of recursive multi-step prediction output based on neural network shown in equation 1:

$$
y_{\mathrm{c}}(k+i \mid k)=y_{\mathrm{m}}(k+i \mid k)+\varphi(\boldsymbol{Y}, \boldsymbol{U}, N N), i=1,2, \cdots p
$$

Where $\boldsymbol{Y}, \boldsymbol{U}$ are past control input values, $N N$ is neural network model, $p$ is the number of predict steps, $y_{\mathrm{c}}(k+i \mid k)$ is output after corrected, $y_{\mathrm{m}}(k+i \mid k)$ is direct output of neural network predict model, $\boldsymbol{\varphi}(\boldsymbol{Y}, \boldsymbol{U}, N N)$ is item of correction function, $\boldsymbol{Y}=[y(k), y(k-1), \cdots] \quad$ are past time output measurement values, $\boldsymbol{U}=[u(k), u(k-1), \cdots]$ are past time input values. Obviously, the choice of $\varphi$ is crucial, which will affect the control performance.

At present, the commonly used method of feedback correction is $\varphi(\bullet)=y(k)-y_{\mathrm{m}}(k \mid k-1)$, this part feedback correction represented as:

$$
y_{\mathrm{c}}(k+i \mid k)=y_{\mathrm{m}}(k+i \mid k)+\left(y(k)-y_{\mathrm{m}}(k \mid k-1)\right)
$$

Where $y(k)$ represents the output measurement of time $k, y_{\mathrm{m}}(k \mid k-1)$ represents $k$ time predicted value based on $k-1$ time.

\section{FEEDBACK CORRECTION METHOD BASED ON NEURAL NETWORK}

\section{A. The Shortcomings of Common Feedback Correction Method}

From the above mentioned can be seen, regular feedback correction is used the previous time compensation error to correct predictive value. But in most cases, there is a big variation difference between $y(k)$ and $y_{\mathrm{m}}(k \mid k-1)$, it is difficult to accurately compensate for model error, it is necessary to find a new method of error compensation $[4,5]$.

\section{B. Improved Feedback Correction Method}

Since the predictive control has been created, people have done a lot of research works about feedback correction, and also achieved some results. 
a) The use of feedback filter for feedback correction of the DMC (Dynamic Matrix Control) algorithm, and DMC algorithm gives a simplified dynamic matrix model[6,7], and based on time series error prediction to rolling optimize system, has good control effect.

b) GPC (the generalized predictive control) algorithm[8], using fuzzy reasoning method for the output error caused by environmental disturbances or model mismatch, proposed the integration of the two control strategies, obtained control the amount by the GPC algorithm, and through fuzzy reasoning get the error step of control amount.

c) Through an in-depth study for predictive functional control algorithm, using fuzzy reasoning method for possible model mismatch situation, then find a fuzzy control volume as compensation volume, and compound added with predictive functional control to get process control inputs volume, it is also an effective way $[9,10]$.

There are other ways to improve forecasting, dynamic generalized method of combining the matrix, multiple feedback methods, cascade control, control weight, have been conducted in-depth research.

\section{Feedback Error Correction Method Based on Neural Network}

In this section, combination with neural network proposed a new compensation correction method based on general error compensation method, the calibration relationship shows as:

$$
y_{\mathrm{c}}(k+i \mid k)=y_{\mathrm{m}}(k+i \mid k)+\varphi\left(\boldsymbol{Y}, \boldsymbol{Y}_{\mathrm{m}}, N N\right) \quad i=1,2, \cdots p
$$

Where $y_{\mathrm{c}}(k+i \mid k)$ is the output value after correct, $y_{\mathrm{m}}(k+i \mid k)$ is the direct prediction output value of neural network model. $\varphi\left(\boldsymbol{Y}, \boldsymbol{Y}_{\mathrm{m}}, N N\right)$ is item of correction function, $\boldsymbol{Y}=[y(k), y(k-1), \cdots]$ are past time output measurement values, $\quad \boldsymbol{Y}_{\mathrm{m}}=\left[y_{\mathrm{m}}(k), y_{\mathrm{m}}(k-1), \cdots\right]$ are past time Predicted output values, $p$ is the number of prediction steps. Use historical error information to construct neural network $\varphi$ about the error $e$, to complete the error compensation correction, making the predicted value closer to the actual value. This calibration relationship fully leverages neural network function approximation ability and generalization ability, since error correction is based on a collection of historical information, which can effectively prevent the impact of uncertainty, to maintain robustness of the system.

Feedback compensation correction algorithms steps based on neural network are as follows:

a) Using neural network prediction model to recursive the predictive value $\boldsymbol{Y}_{\mathrm{m}}$ for a future time.

b) By actual output values of controlled object $\boldsymbol{Y}$ and $\boldsymbol{Y}_{\mathrm{m}}$ to structure a function of prediction error $e_{\text {which based }}$ on feedback neural network model, as the error correction term. c) Using actual predictive value $\mathrm{E}$ for the predictive control, derive Predictive control law, complete the predictive control.

\section{SiMULATION EXAMPLES}

A nonlinear discrete model used for simulation is shown as:

$$
y(k)=\frac{y(k-1)}{1+y^{2}(k-1)}+u(k-1)
$$

\section{A. Expectation Is The Unit Step Signal}

When prediction step is 3 , control field is 2 , expectation is the unit step signal, and simulation results are shown in Fig.1 and Fig.2. Fig.1 shows simulation result of conventional feedback correction control, Fig.2 shows simulation result of feedback correction based on neural network.
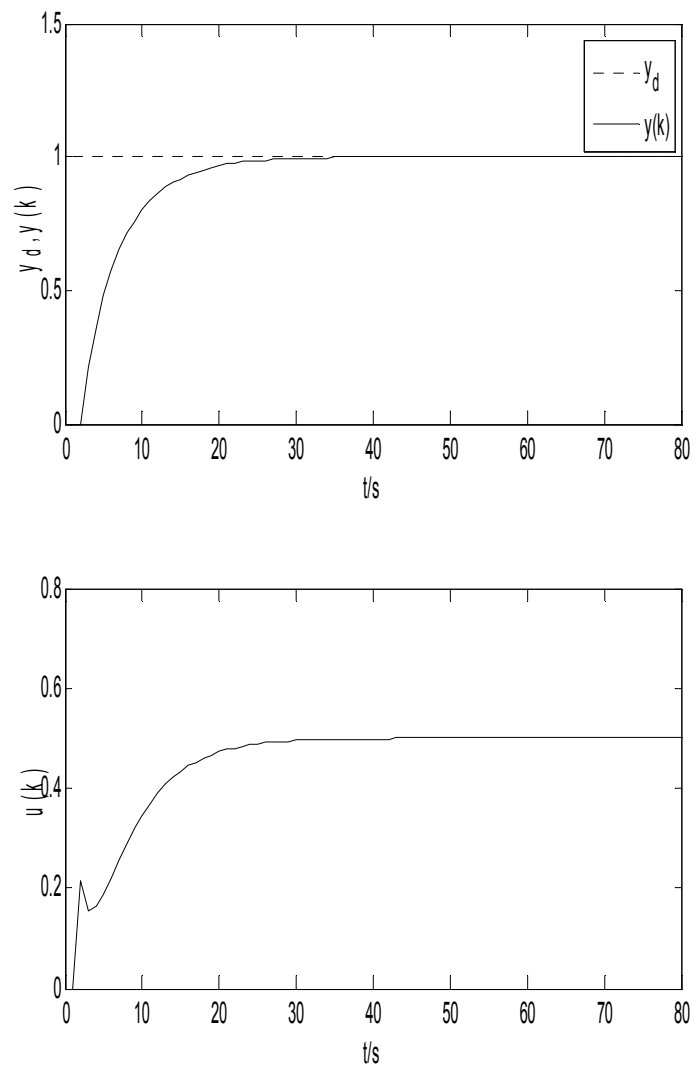

Figure 1 simulation result of conventional feedback correction control

In Fig.1 and Fig. 2, $y_{\mathrm{d}}$ is system Expectation value, $y(k)$ is system Actual output value, $u(k)$ is the control variable, compare two Figures can be seen that neural network error correction can reflect the trend of the error in general, feedback correction by the neural network can speed up response time. 

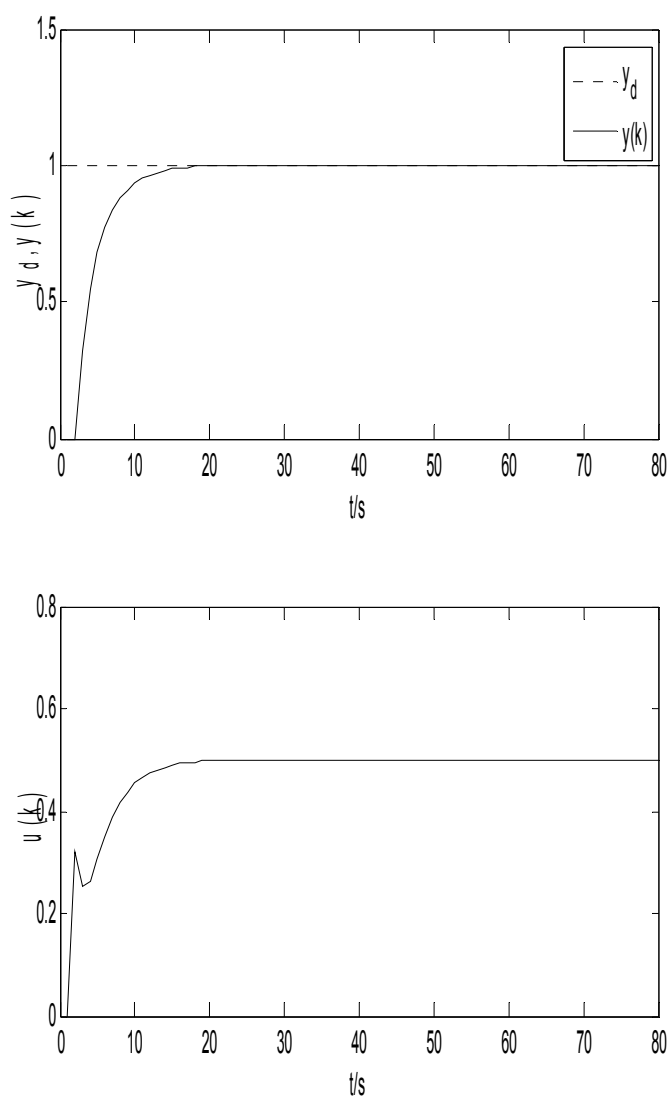

Figure 2 simulation result of feedback correction based on neural network

\section{B. Expectation is The Square Wave Signal Which Amplitude Is 1 .}

Prediction step is 3, control field is 2, and expectation is the square wave signal which amplitude is 1. Fig. 3 shows simulation result of conventional feedback correction control, Fig.4 shows simulation result of feedback correction based on neural network.

When the signal is mutated, neural network error correction can get faster and better compensation for the forecasting error.
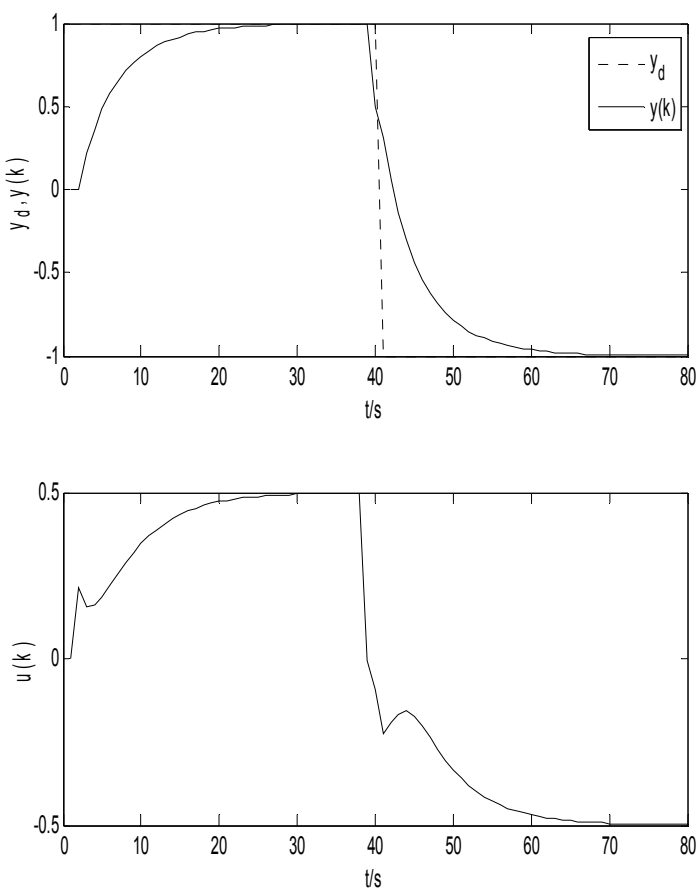

Figure 3 simulation result of conventional feedback correction control
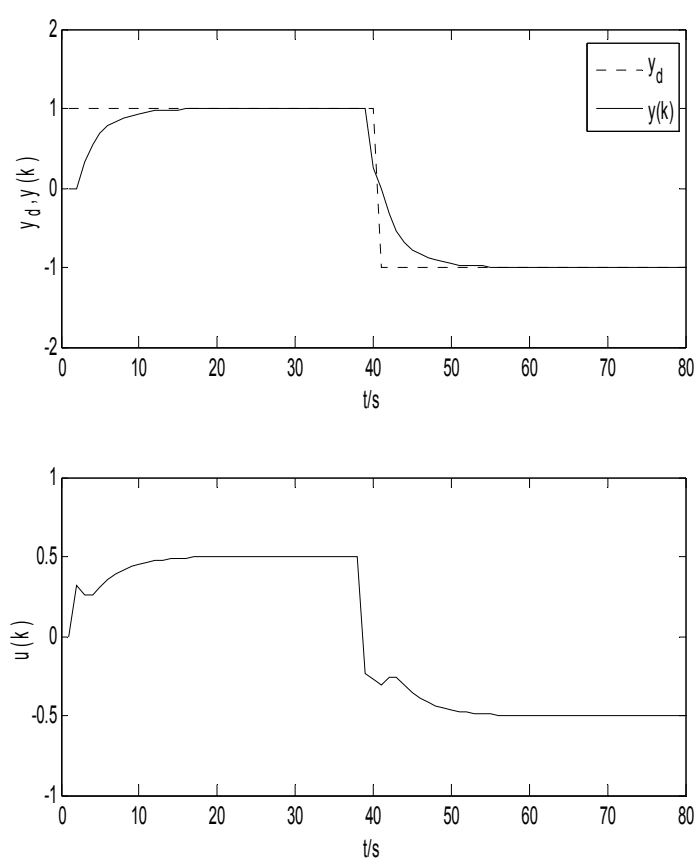

Figure 4 simulation result of feedback correction based on neural network

\section{CONCLUSION}

This paper focuses on neural network-based error feedback correction method, compared with the conventional error feedback correction; Feedback error correction based 
on neural network can be more effective compensation model prediction error and improve the quality of control. The simulation study validates this point.

\section{REFERENCES}

[1] Mcloone S, Brown MD, Irwin G, Light body A. A hybrid linear/nonlinear training algorithm for feedforward neural networks.IEEE Trans Neural Networks 1998; 9(4):669-84.

[2] Narendra K, Parthasarathy K. Identification and control of dynamical systems using neural networks. IEEE Trans Neural Networks 1990; $1(1): 4-27$

[3] Kennedy, J. and Eberhart, R. (1995). Particle Swarm Optimization, IEEE International Conference on Neural Networks (Perth, Australia), IEEE Service Center, Piscataway, NJ, IV: 1942-1948.

[4] Iyoda, E. M., Nobuhara, H., \& Hirota, K. (2003). A solution for the N-bit parity problem using a single multiplicative neuron. Neural Processing Letter, 18, 233-238.
[5] A.E. Eiben, J.E. Smith, Introduction to Evolutionary Computing, Springer, Berlin, 2003

[6] Michalska H,Mayne D Q.Robust receding horizon control of constrained nonlinear systems[J].IEEE Transactions on Automatic Control,1993,38(11):1623-1633.

[7] De Nicolao G,Magni L,Scattolini R.Stabilizing predictive control of nonlinear ARX models[J].Auto- matica,1997,33(9):1691-1697.

[8] Wright, S. J. (1997). Applying new optimization algorithms to modelpredictive control. In: J.C. Kantor, C.E. GarcmHa, B. Carnahan (Eds.),Fifth international conference on chemical process control, CACHE,AICHE(147-155).

[9] Alvarez H,Londono C,Carelli R.PH neutralization process as a benchmark for testing nonlinear controllers[J].Industrial and Engineering Chemistry Research,2001,40(11):2467-2473.

[10] Sarimveis H,Nikolakopoulos A.A line up evolutionary algorithm for solving nonlinear constrained optimization pronlems.Computers and OperationsResearch,2005,32(6):1499-1514. 